\title{
LUCI DELLA CITTÀ. FORME DI ILLUMINAZIONE IN UN CENTRO URBANO MEDIEVALE
}

\section{FRANCESCA ROMANA STASOLLA - GIULIA PREVITI}

UDC: 726(497.54)"12/13"

Original scientific paper

Manuscript received: 12. 11. 2019.

Revised manuscript accepted: 15. 01. 2020.

DOI: $10.1484 /$ J.HAM.5.102674
F. R. Stasolla

G. Previti

Dipartimento di Scienze dell'Antichità Sapienza Università di Roma Piazzale Aldo Moro, 5, 00185 Roma, Italia francescaromana.stasolla@uniromaı.it, xxxx?

The archaeological excavation of town of Cencelle, which began twenty-five years ago, has been an opportunity to reflect on the methods of lighting in a medieval town. In fact, both public and religious neighbourhoods and those with residential and craft functions have been excavated; moreover, documents and archaeological data have allowed the reconstruction of the places where daily life and work took place. In this work the ceramic, metal and glass products connected with instruments intended to shed light are presented, the analysis of which contributes to the verification of the tools used for artificial lighting; in addition, the urban structure and the settlement choices are also analysed, which reflect the light needs of the urban centre. On the subject, Cencelle has no direct written sources, so the material data from the excavation are compared with the historical and documentary data relating to contemporary cities. This type of work contributes to the understanding of the lighting methods of public and private spaces, as well as of the symbolic and ritual value that light had in the conception of a medieval town.

Keywords: Middle Ages, archaeology, methods of lighting, normative sources

A Maria Donatella Gentili ora nella Luce

La ricostruzione del sistema di illuminazione di un centro urbano è di per sé cosa complessa, perché implica la mescolanza di dati materiali, come i manufatti, ed immateriali, come l'orientamento e la disposizione degli edifici, in un intreccio combinato nel quale non è facile capire quali siano i fattori casuali e quali quelli volutamente ricercati. Certamente, gli scavi delle città medievali appaiono connotati dalla scarsa presenza di strumenti da illuminazione, un dato questo su cui certamente pesa la quota di uso di candele, torce e materiali da illuminazione deperibili, dei quali non resta alcun tracciante archeologico. A Cencelle, città dell'alto Lazio fondata alla metà del IX secolo da papa Leone IV e oggetto di una vera e propria riorganizzazione urbanistica tra XII e XIII secolo, 25 anni di ininterrotti scavi archeologici hanno restituto la stessa realtà. Sono stati infatti indagati ambiti diversi della realtà urbana: l'area del potere, con il palazzo pubblico; la sede della guarnizione militare; le aree abitative ed artigianali; gli snodi stradali; il complesso episcopale altomedievale, poi sede della canonica romanica, con annesso cimitero'(Fig. 1). Ambiti diversificati, appunto, che hanno consentito di indagati aspetti diversi della vita cittadina e di acquisire informazioni e manufatti tali da comprendere varie sfaccettature della vita quotidiana. La scarsità degli elementi collegati all'illuminazione ha condotto ad indagare le fonti normative per comprendere quanto di tale mancanza fosse da attribuire alla semplice lacunosità archeologica. La città non dispone di fonti dirette, di qui il ricorso agli statuti dei centri contermini ed ai centri laziali coevi, analoghi per dimensioni, per strutture economiche e politiche. Rispetto alle città romane a tardoantiche, infatti, nelle città del pieno medioevo cessano quali del tutto gli strumenti di illuminazione in ceramica, mentre una modestra presenza è riservata a quelli in metallo ed in vetro, materiali soggetti però a forte riciclo ${ }^{2}$; rimane indeterminabile il peso dei manufatti in materiale deribile, come cera ed essenze resinose ${ }^{3}$.

Veniamo prima alla illuminazione naturale. Appare chiaro come la disposizione topografica della città non sia influenzata dalla disposizione rispetto alla luce solare, quanto piuttosto dalla necessità di rispetto della viabilità preesistente, e lo stesso sfruttamento dell'orientamento favorevole di alcune strade viene meno a causa nella loro ristrettezza, che implica zone d'ombra soprattutto ai piani bassi delle abitazioni. In sostanza, la luce naturale circolava in città, ma la città non era costruita secondo parametri di sfruttamento ottimale della luce, il cui utilizzo viene sub-

\footnotetext{
${ }^{1}$ Per la ricerca archeologica nella città si rimanda a F.R. STASOLLA, Leopoli-Cencelle. Il quartiere sud-orientale, Spoleto 2012; Forma e vita di uan città medievale. Leopoli-Cencelle. Catalogo della mostra, a cura di L. ERMINI PANI, M.C. SOMMA, F.R. STASOLLA, Spoleto 2014; M.C. SOMMA, F.R. STASOLLA, Città fondata e "rifondate": Leopoli-Cencelle, in "Fondare" tra antichità e medioevo. Atti del Convegno di studio (Bologna, 27-29 maggio 2015), a cura di P. GALETTI, Spoleto 2016, pp. 27-43; M.D. GENTILI, M.C. SOMMA, F.R. STASOLLA, Ad locum optimum valdeque munitum: nuovi dati sulla fondazione di Leopoli-Cencelle, in Rendiconti della Pontificia Accademia Romana di Archeologia, 89 (2016-2017), pp. 367-406.

${ }^{2}$ Per una prima valutazione delle presenze percentuali dei diversi materiali si rimanda a F.R. STASOLLA, La portata del deposito: organizzazione sociale e dati quantitativi a Cencelle, in Atti VIII Congresso Nazionale di Archeologia Medievale (Matera, 12-15 settembre 2018), Firenze 2018a, pp. 176-179; F.R. STASOLLA, La ceramica di Cencelle nel medioevo. Alcune riflessioni di metodo per una questione ancora aperta, in Scienze dell'Antichità, 24.1 (2018b), pp. 175-181. ${ }_{3}^{3} \mathrm{Si}$ rimanda a F.R. STASOLLA, Dal tramonto all'alba: strumenti e tecniche di illuminazione nell'alto medioevo, in Il fuoco nell'alto medioevo. Atti della LX Settimana di studio CISAM (Spoleto, 12-17 aprile 2012), Spoleto 2013, pp. 857-888.
} 


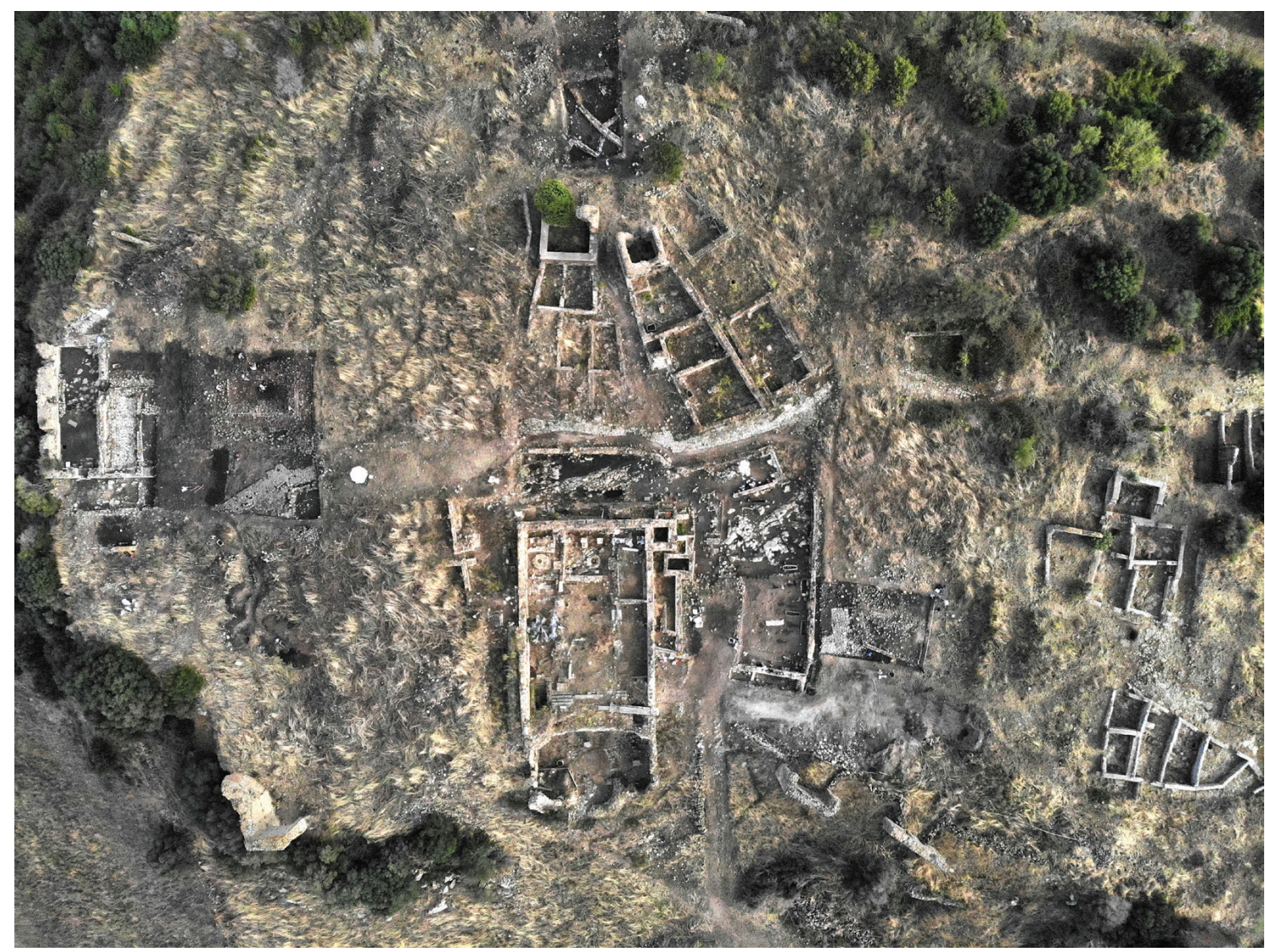

Fig 1: Cencelle, veduta delle aree indagate

ordinato ad altri fattori considerati più rilavanti, come la viabilità, o le esigenze topografiche o difensive.

A questi elementi si aggiungono interessanti aspetti normativi. Infatti, a ben guardare gli statuti dei centri laziali vicini e di quelli di medie dimensioni e di composizione sociale agricolo-artigianale, sui quali quindi Cencelle si può rispecchiare, colpisce una serie di clausole che ha pesanti influenze sulla illuminazione. Appare infatti molto chiaro come dopo la campana che batte il vespro, cioè dopo il convenzionale tramonto, la maggior parte delle attività si fermi, con alcune eccezioni, come ad esempio le fornaci ceramiche, che possono essere accese solo dopo quest'ora ${ }^{4}$. Per il resto, è necessario che ciascuno ritorni a casa e soprattutto e che ci resti. Anche osti e tavernieri devono cessare di mescere vino e di permettere i giochi e devono rimandare a casa gli avventori ${ }^{5}$. Chiunque ha necessità di uscire per giustificato motivo, ad esempio in caso di malattia, non può allontanarsi che di tre case oltre la propria e solo se dotato di un lume almeno ogni tre persone, così da poter essere facilmente riconosciuto ${ }^{6}$. Proprio la stabilità sociale sembra essere alla

\footnotetext{
${ }^{4}$ Lo statuto di Viterbo nella redazione della prima metà del XIII secolo, ad esempio, prevede che: De pena pulsantis aliquam campanam ad rumorem sine licentia potestate. Siquis propter aliquem rumorem pulsaverit aliquam campanam sine licentia potestatis, puniatur udque in XXV libras (Statuti della provincia romana. S. Andrea in Selci, Subiaco, Viterbo, Roviano, Anagni, Saccomuro, Aspra Sabina, ed. R. MORGHEN, P. EGIDI, A. DIVIZIANI, O. MONTENOVESI, F. TOMMASETTI, P. FONTANA, a cura di V. FEDERICI, Roma 1930, 1251-2 IIII Maleficiorum [XXI], p. 223); poco più avanti aggiunge poi: De pena figuli mictentis ignem ad vasa coquenda ante vesperas. Volumus quod nulli figulo vel vascellario liceat ignem mictere ad vasa coquenda nisi post vesperas, et qui contrafecerit, in XX solidis per Curia puniatur (1251-2 III Maleficiorum [XXVII], p. 224).

${ }^{5}$ Così ad esempio negli statuti di Viterbo: Quod tabernarii non permictant aliquem ludere in taberna, et quod non substineant aliquem ludum post primam campanam. Tabernarii, vel qui vinum vendunt, non permictant aliquem ludere in taberna aluquo ludo unde acquiratur aliquid vel perdatur, et post primam pulsationem campane S. Angeli aliquem non substineant nec permictant in tabernis suis morari, nec postea recipiant venientes ad bibendum vel comedendum; et si contrafacntum fuerit, tabernarius, vel qui vinum vendit, solvat Curie IX solidos; et qui inventus fuerit ibi bibere vel ludere, CC solidos solvat pro pena; et hoc ibidem, si prope tabernam post campanam luxerit vel potaverit, observetur; quam penam non solvat, qui miserit aliquam personam ad tabernam aliquam ad vinum sibi emendum, dum tamen lumen portet in manu, nec persona missa taliter teneatur as penan; Statuti della provincia romana. S. Andrea in Selci, op. cit. (n. 3), pp. 242-243 [C]. La stessa prescrizione ricorre negli statuti di Roccantica del 1326 (Statuti della provincia romana. Vicovaro, Cave, Roccantica, Ripi, Genazzano, Tivoli, Castel Fiorentino, a cura di F. TOMASSETTI, V. FEDERICI, P. EGIDI, Roma 1910, p. 89), in quelli di Ripi del 1331 (Statuti della provincia romana. Vicovaro, op. cit. (supra), p. 117), nello Statuto di Tivoli del 1305 (Statuti della provincia romana. Vicovaro, op. cit. (supra), pp. 187-188), nello statuto di Alatri (Gli statuti medioevali del castello di Alatri, a cura di M. D’ALATRI, C. CAROSI, Roma 1976, p. 184).

${ }^{6}$ Queste le prescrizioni nello statuto di Viterbo: De pena euntis post tertium sonum campane. Statuimus post tertiam pulsationem campane constitute nullus male fame per Civitatem vel extra, aliquo modo vel ingenio, audeat ire; et si inventus fuerit, ducatur ad potestatem vel consulem, et in C solidis puniatur. si vero in maleficio fuerit inventus, puniatur arbitrio potestatis vel consulis. si vero bone fame fuerit inventus, puniatur in XX solidis. si autem ab agro vel a vinea vel ab alio loco reddierit et sine lumine, liceat ei ad domum redire sine pena; et de hoc credatur sacramento eius, si fueritur bone fame. item cum lumine possit aliquis ire sine pena; quod obtineat, si unus cum plurimus simul portaverit lumen accensum; et de lumine, quod portaverit, stetur suo iuramento, si est homo bone fame. et si homo bone fame ante vel iusta domum suam vel per tres domos a longe a domo sua fuerit inventus, no patiatur pena; Statuti della provincia romana, op. cit. (n. 3), p. 250, 1251-2 [CXXVIII]. Questa norma compare anche nello statuto di Cave, nella versione del 1307 (Statuti della provincia romana. Vicovaro, op. cit. (n. 4), pp. 44-45), negli statuti di Ripi, del 1331 (Statuti della provincia romana. Vicovaro, op. cit. (n. 4), p. 116, dove va notato come fossero previste alcune eccezioni per attività evidentemente non dilazionabili). La regola accomuna anche centri del Lazio meridionale, come Alatri (Gli statuti medioevali del castello di Alatri, op. cit. (n. 4), p. 184 e pp. 185-185).
} 
base di questa regola ferrea, che accomuna tutti gli statuti del Lazio medievale e che spinge a raddoppiare le sanzioni per tutti i reati compiuti di notte ${ }^{7}$, e che si muove in linea con altre forme di controllo sociale, come quelle che regolano le adunate, il numero di persone che possono partecipare ai funerali, le leggi suntuarie ${ }^{8}$. Il divieto di circolazione viene meno solo per casi straordinari, come il trasporto del mosto nel corso della vendemmia 9 . Anche le attività collegate con l'agricoltura e con il passaggio degli animali si fermano ${ }^{10}$. Una città quindi che al calar del sole è priva di illuminazione pubblica e che è sostanzialmente a buio, soggetta ad una sorta di coprifuoco; i suoi abitanti restano a casa, o nelle corti interne alle abitazioni, che sono illuminate da fuochi liberi ${ }^{11}$.

In queste città buie e spente, momenti di vero splendore dovevano essere rappresentati dalle feste liturgiche più importanti, tutte significativamente collegate all'accensione di lampade. La notte di Pasqua, in particolare, rappresenta l'esplosione della luce, il momento in cui il valore simbolico doveva sommarsi ad una vera e propria esplosione di luce, destinata a sublimare l'evento e l'edificio stesso.

Francesca Romana Stasolla

Gli strumenti per l'illuminazione costituiscono una categoria di manufatti fortemente legata alla sfera del quotidiano. Generalmente, se composti da materiali non deperibili, risultano però scarsamente documentati sotto il profilo archeologico e ancor meno nelle fonti documentarie. A ciò si aggiunge poi un naturale squilibrio numerico legato all'alto tasso quantitativo dei reperti ceramici a fronte del vetro e del metallo, soggetti a forme più intense di riuso già in antico. Oltre che la mancata trasmissione di oggetti da illuminazione, ottenuta spesso grazie ai soli combustibili, come nel caso delle candele e delle torce.

Per tutto l'altomedioevo l'illuminazione si avvale di combustibili liquidi, come olii animali e vegetali, a cui si affiancano anche i combustibili solidi, come sego e cera per la realizzazione delle candele. Queste ultime andarono di frequente quasi a sostituire, in età bassomedievale, le lampade ad olio, determinando anche delle scelte funzionali nell'uso del contenitore. Dal VI secolo, compaiono infatti sul mercato le lucerne cosiddette aperte, che continueranno per tutto il medioevo e che testimoniano proprio la diffusione di combustibili solidi che si affiancano a quelli liquidi ${ }^{12}$. Le candele tuttavia sono più sfuggenti dal punto di vista della ricostruzione archeologica, in quanto destinate a consumarsi nell'uso, anche se le fonti scritte ed iconografiche ci possono offrire un quadro realistico dei modi di utilizzo, da sole o combinate con altri supporti. Esse potevano poggiare su oggetti di materiale diverso, realizzati per ospitare o singole candele o un numero molto più ampio, se parliamo di manufatti più complessi.

I ritrovamenti materiali registrati nella città di Cencelle in questi anni di intensa attività di scavo, in parte confermano questa tendenza e in parte la ribaltano. Se analizziamo infatti il numero di reperti riferibili all'illuminazione, noteremo una totale assenza di manufatti ceramici, che lascia spazio invece alla presenza di materiale metallico e, in piccolissima parte, anche vitreo.

Certamente entra in gioco un fattore di non riconoscibilità dei supporti ceramici, poiché oltre alla classica lucerna, altre forme più semplici e largamente utilizzate nel quotidiano, come bugie e piattini, possono assolvere alla stessa funzione in presenza di combustibili solidi, per i quali è anche difficile distinguerne le tracce d'uso nel tempo. Non è da escludere inoltre anche l'utilizzo di supporti lignei o che candele e ceri venissero posti direttamente su piani di appoggio come tavoli o mensole. Contestualmente una minore attestazione di elementi ceramici nei secoli finali del medioevo, attestata in larga parte in Toscana e nelle regioni del sud Italia, può ragionevolmente essere spiegata con un uso sempre più diffuso di vetro e metallo anche in classi meno agiate.

I contesti di rinvenimento sono per lo più afferenti ai quartieri residenziali e artigianali collocati a sud-est della città,

\footnotetext{
${ }_{7}^{7}$ Così è prescritto nello statuto di Roccantica: [LIIII] De incendiis ponendis in area seu aliquo genere bladi choadunati in area seu campo. Item, statuimus et ordinamus quod quicumque incendium posuerit in area seu in aliquo genere blai choadunati in area seu campo, in spicis videlicet grani, ordei, milei, spelte, secle, farris, panici, sagine vel in alique genere leguminum, puniatur vice qualibet in vigintiquinque libris provisinorum, et nichilominus ad emendationem dampni; et si de nocte fuerit positum ipsum incendium delinquens puniatur in pena dupli superius denotata, et in eadem pena puniatur quicumque posuerit incendium extra castrum predictum... ; Statuti della provincia romana. Vicovaro, op. cit. (n. 4), p. 76. Anche a Ripi si prevede il raddoppio delle pene per i reati notturni (Statuti della provincia romana. Vicovaro, op. cit. (n. 4), p. 121), così nello Statuto di Tivoli del 1305 (Statuti della provincia romana. Vicovaro, op. cit. (n. 4), p. 209), negli Statuti di Castel Fiorentino, le cui norme vengono riproposte sia nelle redazioni del 1298 che in quelle del 1305 (Statuti della provincia romana. Vicovaro, op. cit. (n. 4), pp. 319 e 322), negli Statuti di Alatri (Gli statuti medioevali del castello di Alatri, op. cit. (n. 4), p. 1859, nello Statuto del castello di Tecchiena, compilato tra il 1372 ed il 1373 (Gli statuti medioevali del castello di Tecchiena, a cura di M. D'ALATRI, C. CAROSI, Roma 1976, p. 75). ${ }^{8}$ La morte e i suoi riti in Italia tra Medioevo e prima età moderna, a cura di F. SALVESTRINI, G.M. VARANINI, A. ZANGARINI, Firenze 2007. Proprio in riferimento a Cencelle era stata condotta una prima analisi F.R. STASOLLA, Chiesa e sepolture: simbiosi e alterità in una città di fondazione tra IX e XV secolo, in Hortus Artium Medievalium, 24, 2019, pp. 555-562.

${ }^{9}$ Così viene, ad esempio, espressamente previsto dallo statuto di Tivoli del 1305: [CLXXXV] Pena euntium post tertium sonum campane. Statuimus quod nullus eat per civitatem Tyburis post tertium sonum campane sine licentia curie vel capudmilitie. Et qui contrafecerit, nisi haberet lumen, et cum uno lumen [...] igne possint ire tres persone, solvat pro banno curie sollidos provisinorum quinque, excepto quod tempore vindemiarum et messium inventus non taneatru ed penam, nisi esset persona male fame et levis vite, et salvo quod vecturales in faciendo et portando vinum et alias res et euntes et redeuntes extra civitatem et per civitatem pro evidenti necessitate, et qui inventi essent in nocte Natalis Domini et diei veneris sancti et in nocte sancte Marie de agusto et beati Laurenti, vel qui inventi ed tres domos convicinas proximas sibi coniunctas ispi domi, non teneatur ad penam; Statuti della provincia romana. Vicovaro, op. cit. (n. 4), pp. 214-215.

${ }^{10}$ Dallo statuto di Ripi del 1331: [LX] Item, quicumque, post ultimum sonum scarane, moram in campo traherit cum bestiis vel sine puniatur in solidis X et ad hoc bannum non teneatur pecodarii. [LXI] Item, quod pecudarii non debeant noctis tempore morari inter esbarras dicti castri cum eorum bestiis a kalendis agusti usque ad festum Omnium Sanctorum, et nihilominus mictere oves per magesias III diebus post pluviam; et qui contra fecerit puniatur in solidis III; Statuti della provincia romana. Vicovaro, op. cit. (n. 4), p. 121.

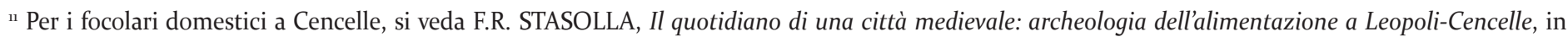
Le archeologie di Merilli. Miscellanea di studi in ricordo di Maria Maddalena Negro Ponzi Mancini, a cura di P. DE VINGO, Alessandria 2018 , pp. 511-522.

${ }^{12}$ STASOLLA 2013, op. cit. (n. 3), pp. 860-867.
} 
1
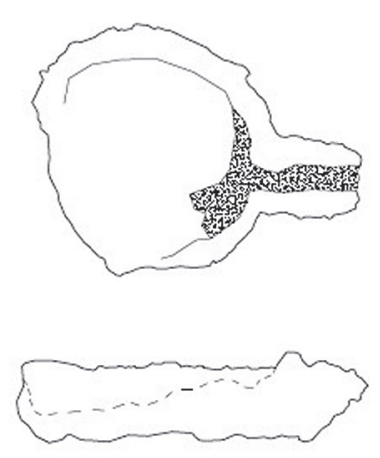

2
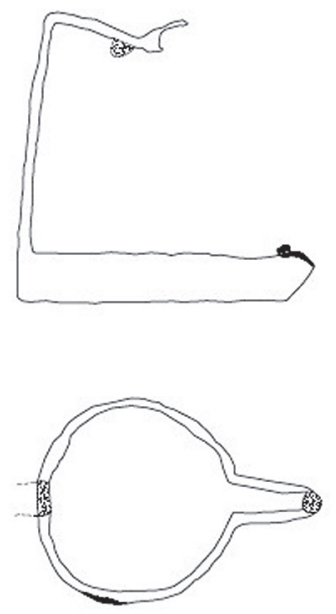

3

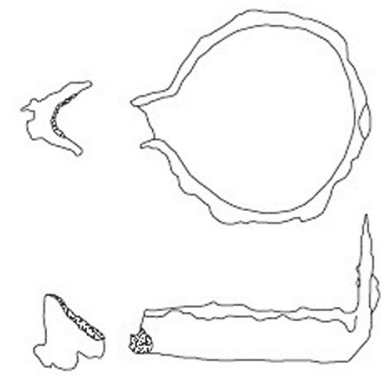

delle case ad un solo ingresso, ben noto nel comparto laziale ed ancora apprezzabile in molti centri a continuità di vita'3.

I manufatti in metallo, che nella seguente trattazione risultano di gran lunga quelli predominanti, attestano una netta prevalenza dell'uso del ferro e in generale ci restituiscono un arco cronologico che va dal XIII al XV secolo. La loro importanza a Cencelle è resa evidente dall'ampio numero di manufatti rinvenuti, ma anche dall'individuazione di scarti di lavorazione, di materie prime, di strutture connesse alla lavorazione del ferro, avvalorata dalla menzione, in un trattato di alleanza tra Cencelle e Viterbo del 1220, di alcuni fabbri nel consiglio della città ${ }^{14}$.

I reperti metallici da illuminazione Scala 1:3

Fig. 2: Lucerne provenienti dalla città di Cencelle: 1. Lucerna a serbatoio aperto con canale rettangolare; 2-3. Lucerne a serbatoio aperto dotate di stelo atto alla sospensione.

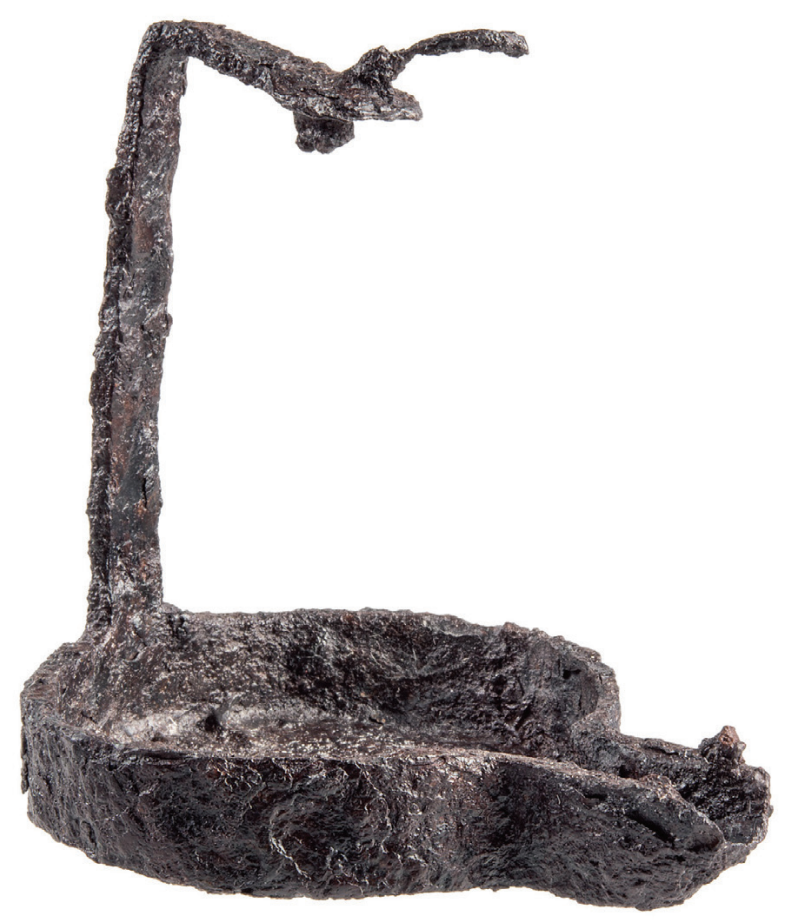

Fig. 3: Lucerna a stelo proveniente da Cencelle

composti da una serie di case-bottega a schiera organizzate per lotti modulari, che si installano su vie di collegamento interne alla città. Un modello abitativo che riprende quello pensione, raccordi e catene, per i quali motivo di incertezza risulta l'impiego indifferente per altre classi di manufatti metallici a sospensione come incensieri, stadere o materiali legati all'edilizia. Le tipologie rinvenute sono abbastanza standardizzate: 5 lucerne a serbatoio aperto di forma circolare; 1 portacandele a stelo e 1 bugia.

Cominciando dall'analisi dei manufatti più numerosi, notiamo che le lucerne si presentano come elementi semplificati rispetto ai confronti tipologici riscontrati sia in Italia che in Francia per lo stesso periodo. Questi ultimi sono caratterizzati dalla presenza di più canali e beccucci rettangolari che fornivano molteplici punti di fuoco, probabilmente legati all'esigenza di illuminare ambienti di maggiori dimensioni ${ }^{15}$. Le lucerne prese in esame si compongono invece di un serbatoio largo $8 \mathrm{~cm}$, con un solo canale rettangolare lungo circa 3,5 cm e largo 2,5 cm ed è possibile datarle tra la metà del XIV e il XV secolo. Due di esse sono accomunate dalla presenza di uno stelo atto alla sospensione, posto nella parte opposta rispetto al beccuccio, una di queste risulta frammentaria nella parte superiore, mentre la lunghezza dello stelo nell'esemplare integro è di circa $10 \mathrm{~cm}^{16}$ (Figg. 2-3).

Un supporto fondamentale alla visualizzazione e ricostruzione grafica dei manufatti in questione, oltre che alla loro messa in opera, ci giunge dalle fonti iconografiche. Un rapido esame dell'iconografia medievale conferma la presenza di oggetti per l'illuminazione in rappresentazioni riguardanti ambienti domestici e vita quotidiana, anche a

\footnotetext{
13 Per un approfondimento sulle stratigrafie provenienti dai settori abitativi di Cencelle: STASOLLA 2012, op. cit. (n. 1) pp. 123-295.

${ }^{14}$ Per un quadro più chiaro degli aspetti sociali della città: F.R. STASOLLA, L'assetto sociale della città, in Forma e vita di una città medievale. Leopoli-Cencelle. Catalogo della mostra, a cura di L. ERMINI PANI, M.C. SOMMA, F.R. STASOLLA, Spoleto 2014, p. 79; STASOLLA 2012 , op. cit. (n. 1) pp. 94-122. Per un approfondimento sulla produzione del metallo e sui materiali editi: V. LA SALVIA, La produzione del metallo, in Forma e vita di una città medievale. Leopoli-Cencelle. Catalogo della mostra, a cura di L. ERMINI PANI, M.C. SOMMA, F.R. STASOLLA, Spoleto 2014, pp. 87-89; V. LA SALVIA, I fabbri di Cencelle, in Forma e vita di una città medievale. Leopoli-Cencelle. Catalogo della mostra, a cura di L. ERMINI PANI, M.C. SOMMA, F.R. STASOLLA, Spoleto 2014, pp. 87-89; R. MARTORELLI, Settore I, in Leopoli-Cencelle. Il quartiere sud-orientale, a cura di F.R. STASOLLA, Spoleto 2012, pp. 134-141; F. ZAGARI, I metalli, in Leopoli-Cencelle. Il quartiere sud-orientale, a cura di F.R. STASOLLA, Spoleto 2012, pp. 252-273.

${ }_{15}$ Si vedano i confronti in D. D'ARCHIMBAU, Les Fouilles de Rougiers: contribution à l'archéologie de l'habitat rural médiéval en pays méditerranéen, Parigi 1980, pp. 1157-1159; F. SOGLIANI, Utensili, armi e ornamenti di età medievale da Montale e Gorzano, Modena 1995, pp. 74-75.

${ }^{16}$ Esempi di questo tipo sono ben rappresentati nelle collezioni private dei musei francesi, nello specifico si vedano i reperti del Musée Carnavalet. H. D’ALLEMAGNE, Histoire du luminaire depuis l'époque romaine jusqu'au XIXe siècle, Paris 1981, pp. 192-193, 237-238.
} 


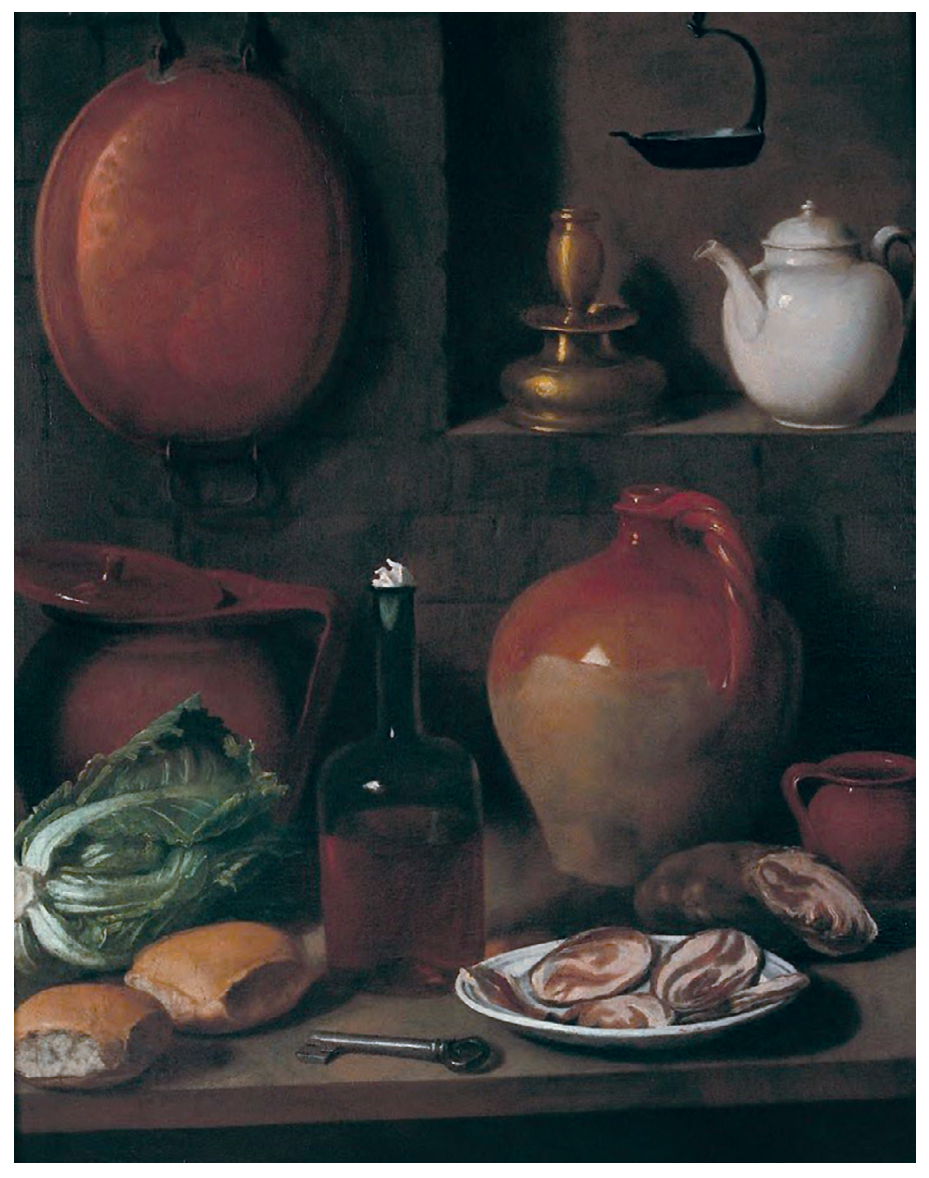

Fig. 4: Carlo Magini, Natura morta con pane, bottiglia di vino e piatto con affettato. Olio su tela; cm. 78x62. Acquisto della Cassa di Risparmio di Fano, 1983.

1
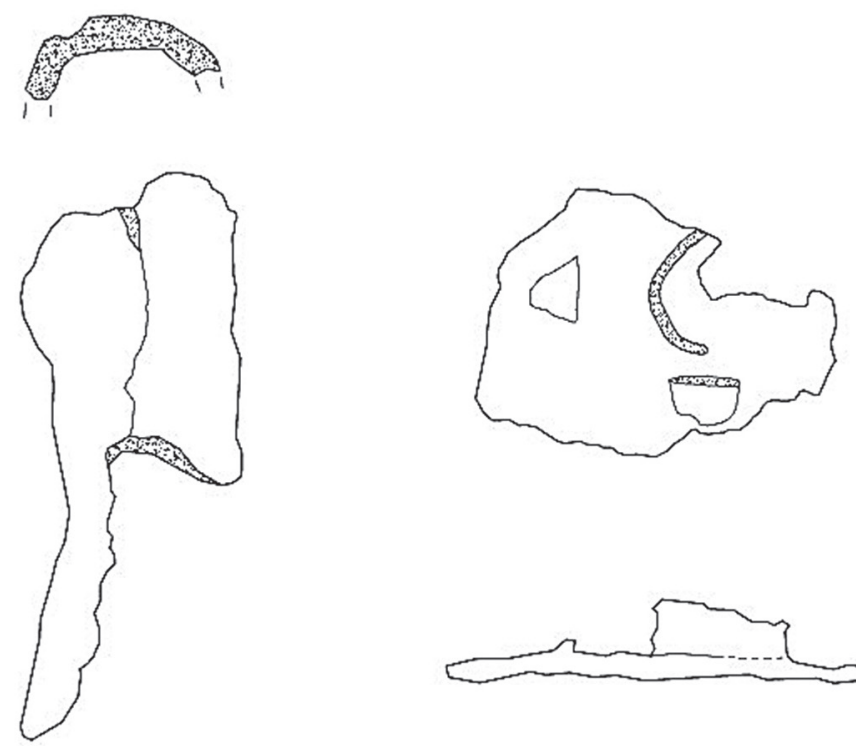

\section{Scala 1:2}

Fig. 6: Portacandele a stelo proveniente da Cencelle; 2. Bugia in metallo che presenta lamina piatta e anello centrale atto ad ospitare la candela.

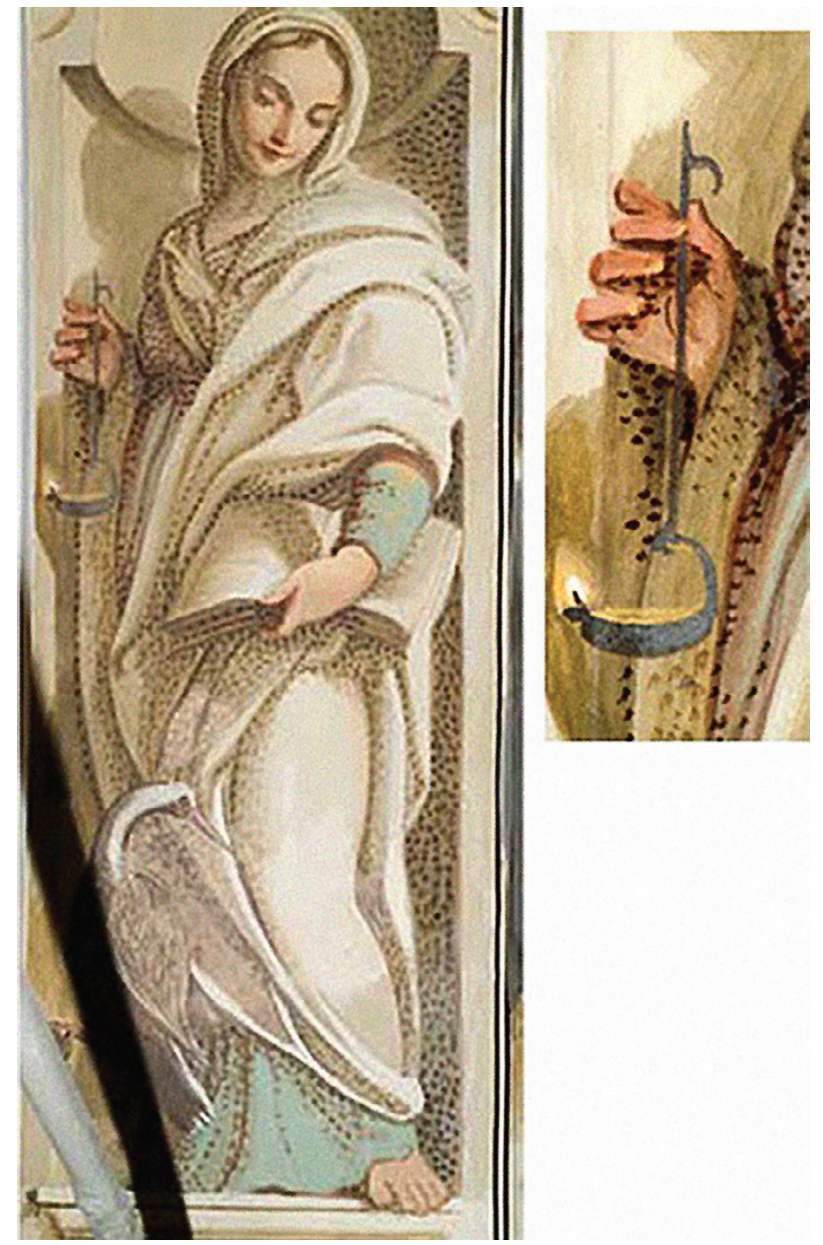

Fig. 5: Raffigurazione della Vigilantia che reca in mano una lucerna, dagli affreschi che decorano la cupola dell'Abbazia di Santa Maria del Monte a Cesena.

seguito di scene tratte dalla Bibbia, e in ambiti religiosi legati alla ritualità cristiana. In questo caso ad esempio ci troviamo di fronte a due scenari molto diversi anche nel tempo, in cui vediamo raffigurato lo stesso tipo di lucerna pensile in metallo in un particolare della tela Natura morta con pane, bottiglia di vino e piatto con affettato di Carlo Magini (1740-1806) ${ }^{17}$ e allo stesso modo in una raffigurazione della Vigilantia che reca in mano una lucerna, dagli affreschi che decorano la cupola dell'Abbazia di Santa Maria del Monte a Cesena, XVIII secolo ${ }^{18}$ (Figg. 4-5).

Proseguendo nella trattazione dei manufatti provenienti da Cencelle, la presenza di un portacandele a stelo rientra anch'essa nella periodizzazione precedente, poiché questi elementi sono attestati in tutta Europa e soprattutto in Francia, a partire dalla metà del XIII secolo fino al XV secolo. Si tratta di manufatti in ferro, composti da un fusto appuntito (da fissare su legno o pietra), a cui si lega una parte superiore di forma circolare ottenuta da una o più estremità ripiegate a formare un manicotto che tratteneva la candela. L'individuo in questione presenta un diametro circolare di circa 4 $\mathrm{cm}$ a cui si lega un fusto frammentario di $6 \mathrm{~cm}$. (Fig. $6, \mathrm{n}$. 1) Trova confronti con esemplari provenienti dagli scavi di Rougiers $^{19}$, da Castel San Pietro in Canton Ticino ${ }^{20}$, dalla

${ }^{17}$ D. DIOTALLEVI, Le nature morte della Quadreria, Fondazione Cassa di risparmio di Fano 2015, pp. 10-11.

${ }^{18}$ F. FARANDA, La Cupola dell'Abbazia di Santa Maria del Monte a Cesena, Cesena 2009, pp. 58-60.

${ }^{19}$ D. D’ARCHIMBAU, Les Fouilles de Rougiers: contribution à l'archéologie de l'habitat rural médiéval en pays méditerranéen, Parigi 1980, pp. 477-478, figg. p. 454 n. 8-9, p. 457 n. 5-6.

${ }^{20}$ A. MARTINELLI, C. DE MICHELI, J. MAY, Indagine archeologica sulla collina di S. Pietro nel comune di Castel S. Pietro (Canton Ticino), in Archeologia Medievale, XXIII (1996), pp. 197-199, tav. II n. 11-12. 


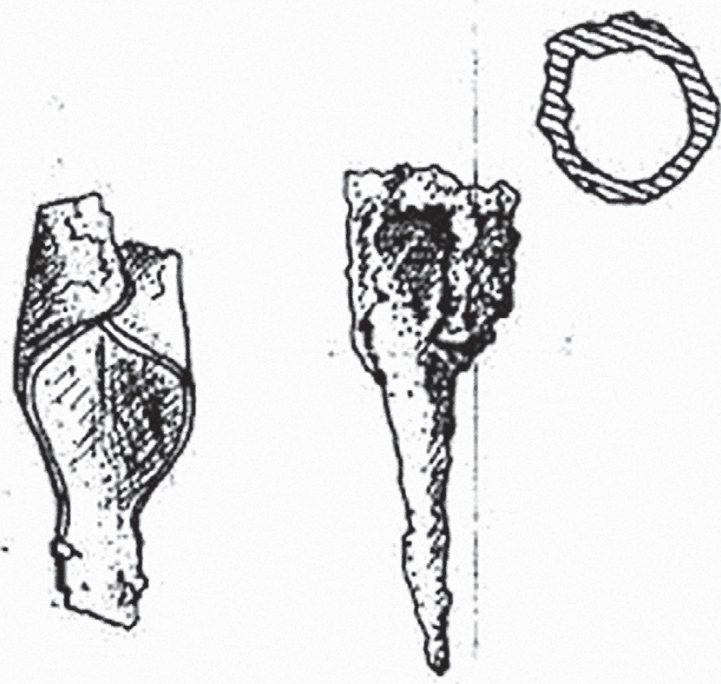

Fig. 7: Portacandele a stelo da Castel San Pietro in Canton Ticino (da A. MARTINELLI, C. DE MICHELI, J. MAY 1996, pp. 197-199, tav. II, 11-12).

fortificazione di Tor dei Pagà a Vione in Valcamonica ${ }^{21}$, da collezioni del Museo Civico di Argenta, in provincia di Ferrara $^{22}$, dal castello di Montaldo di Mondovì in provincia di Cuneo $^{23}$ (Figg. 7-8).

Infine, l'unico esemplare di bugia ritrovato è caratterizzato da una semplicità morfologica che prevede una lamina piatta, conservata per circa $10 \mathrm{~cm}$, di cui non è possibile comprendere i limiti, con anello centrale di diametro pari a $3,5 \mathrm{~cm}$, atto ad ospitare la candela. (Fig. 6, n. 2) Si tratta del tipo base di bugia, con alto carattere funzionale e dunque lunga continuità tipologica. Si trovano esemplari simili da uno strato di discarica del XIV secolo da Castel di Pietra a Gavorrano, da Donoratico e da Rocca San Silvestro in stratigrafie databili alla seconda metà del XIII secolo ${ }^{24}$, da Pisa invece in contesti urbani che ne spingono la datazione fino all'inizio del XIX secolo' 25 (Fig. 9).

Un unico caso isolato, proveniente invece dalle stratigrafie della chiesa bassomedievale di Cencelle, testimonia la presenza di una lampada vitrea pensile di derivazione orientale, di cui è giunta fino a noi solo un'ansa ad applique, con spessore di o,4 cm, larghezza di o,5 $\mathrm{cm}$ e altezza di 1,4 $\mathrm{cm}$. Tali lampade vitree, pur presenti nel periodo romano e

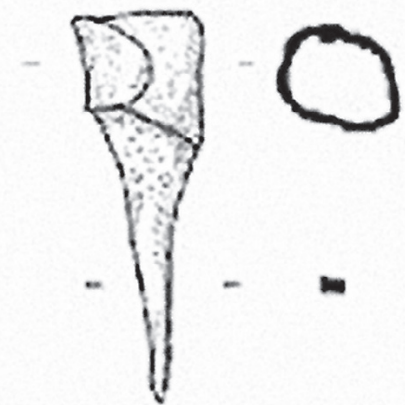

Fig. 8: Portacandele a stelo dalla fortificazione di Tor dei Pagà a Vione in Valcamonica (da G. BELLANDI et alii 2015, pp. 107-108, tav. II n. 32.
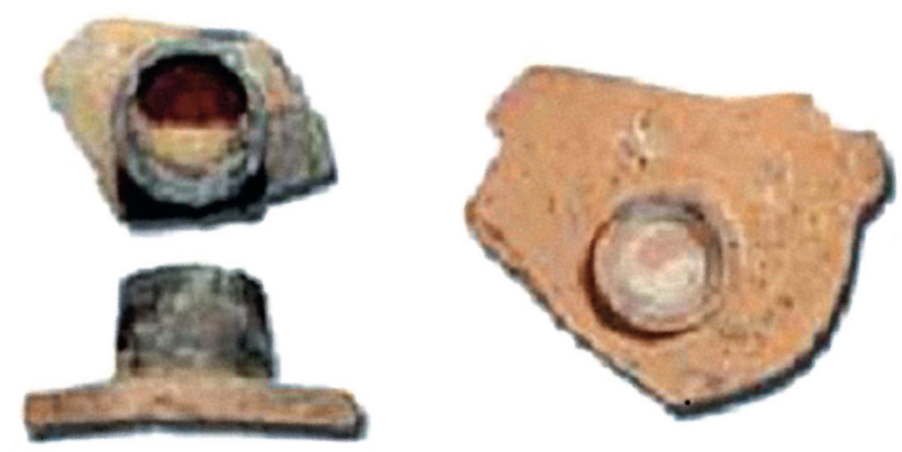

Fig. 9: - Esemplari di bugie in ceramica, provenienti da Pisa in contesti urbani (M. GIORGIO 2017, pp. 248-253, fig. 6, M-N).

tardoantico, diventano predominanti per tutto il medioevo e sono caratterizzate da un alto orlo svasato, piccole anse verticali impostate sul corpo globoso e piede a profilo troncoconico, o più di rado ad anello, la cui introduzione in Italia - a Ragogna (UD) ${ }^{26}$, Castiglione (RI) ${ }^{27}$, Grado (GO) ${ }^{28}$, Santa Giulia di Brescia ${ }^{29}$, Massafra (TA $)^{30}$ - è testimoniata almeno dall' XI secolo, ma è tuttora documentata in prevalenza in stratigrafie bassomedievali, soprattutto tra XIII e XIV secolo. (Fig.. 10) In essa si versa il combustibile, eventualmente galleggiante su acqua, e si inserisce lo stoppino, che viene sostenuto da un reggistoppino di metallo agganciato in genere ai bordi della lampada. Il contenitore viene quindi sospeso mediante catenelle agganciate a delle piccole anse

${ }^{21}$ G. BELLANDI et alii, La fortificazione di Tor dei Pagà a Vione (Valcamonica, BS). Risultati delle Campagne archeologiche 2011-2014, in Archeologia Medievale, XLII (2015), pp. 107-108, tav. II n. 32.

${ }^{22}$ M. LIBRENTI, I metalli, in Il Tardo Medioevo ad Argenta. Lo scavo di via Vinarola-Aleotti, a cura di C. GUARNIERI, Firenze 1999, pp. 124-125, tav. 32 n. 30. ${ }^{23}$ M. CORTELLAZZO, C. LEBOLE di GANGI, I manufatti metallici, in Montaldo di Mondovi. Un insediamento protostorico. Un castello, a cura di E. MICHELETTO, M.V. GAMBARI, Cuneo 1991, pp. 213-214, fig. 119 n. 7.

${ }^{24}$ M. BELLI, Castel di Pietra (Gavorrano - GR): relazione preliminare della campagna 2001 e revisione dei dati delle precedenti, a cura di C. CITTER, in Archeologia Medievale, XXIX (2002), pp. 156-157, tav. 15.

${ }_{25}$ M. GIORGIO, Oggetti per l'illuminazione a Pisa e nella Toscana settentrionale, in Ceramica e vetro nell'illuminazione dal Medioevo alle esperienze nella produzione contemporanea, Atti del XLIX Convegno Internazionale della ceramica Albisola 2016, Savona 2017, pp. 248-253, fig. 6 (M-N).

${ }^{26}$ S. LUSUARDI SIENA, R. ZUECH, Una lampada di tipo islamico dal castrum di Ragona (Udine, Friuli), in Annales du 14e Congrès de l'Association Internationale pour l'Histoire du Verre: Venezia-Milano 1998, pp. 243-247.

${ }^{27}$ F. DEL VECCHIO, Lampade di tipo islamico dal Castellum di Castiglione (Ri), in Il vetro nell'Alto Adriatico. Atti delle IX Giornate Nazionali di Studio (Ferrara, 13-14 dicembre 2003), a cura di D. FERRARI, A.M. VISSER TRAVAGLI, pp. 95-98, fig. 5.

${ }^{28}$ A. MARCANTE, Materiale vitreo da Grado: proprietà Fumolo, Campo Patriarca Eliaı. Rapporto preliminare, in Il vetro nell'Alto Adriatico. Atti delle IX Giornate Nazionali di Studio (Ferrara, 13-14 dicembre 2003), a cura di D. FERRARI, A.M. VISSER TRAVAGLI, p. 52, fig. 1. 9.

${ }^{29} \mathrm{M}$. UBOLDI, Recipienti in vetro di età altomedievale da recenti scavi archeologici in Lombardia, in Annales du 14e Congrès de l'Association Internationale pour l'Histoire du Verre: Venezia-Milano 1998, pp. 208-209, fig. 3 n. 1.

$3^{30}$ R. CAPRARA, I vetri nella ricerca archeologica degli ultimi trent'anni in Puglia e Basilicata, in Quaderni Friulani di Archeologia, XIX (2009), pp. 59-69, fig. 2 n. 2. 


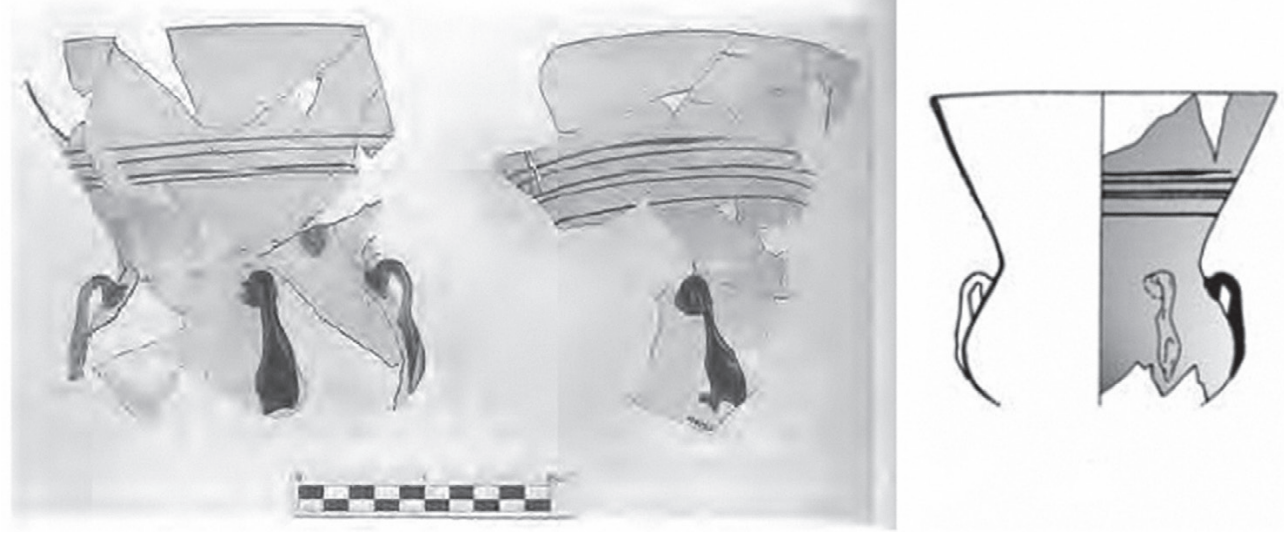

Fig. 10: Lampada di tipo islamico da Ragona (da S. LUSUARDI SIENA, R. ZUECH 1998, p. 244, fig. 2-3)

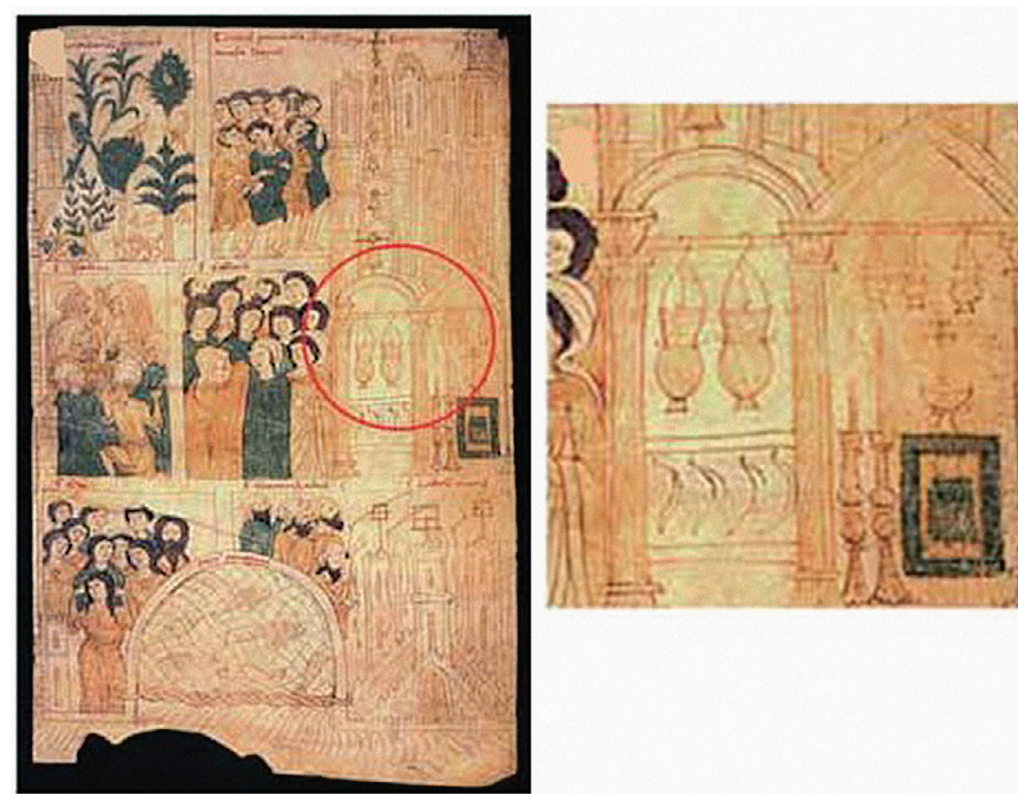

Fig. 11: Miniatura n. 5 del Codice conservato a Berna (Liber ad honorem Augusti sive De Rebus Siculis Carmen di Pietro da Eboli).

poste al di sotto del collo del recipiente, che convogliano in un uncino agganciato al soffitto. Sotto il profilo formale, molte delle lampade vitree sono in realtà assimilabili a bicchieri o recipienti utilizzati in un modo o nell'altro, a prescindere dalla destinazione originaria. Sul piano pratico infatti si aggiunge spesso la difficoltà di riconoscere come pertinenti a suppellettile da illuminazione $i$ frammenti delle parti morfologiche che la lampada ha in comune con altri manufatti vitrei a destinazione diversa o molteplice. A questo proposito un interessante riferimento iconografico si può rintracciare nella miniatura (la n. 5 del Codice conservato a Berna), che illustra il cordoglio della città di Palermo per la morte di Guglielmo II, nel Liber ad honorem Augusti sive De Rebus Siculis Carmen di Pietro da Eboli, scritto tra 1194 e 1197 per celebrare la conquista del regno di Sicilia da parte di Enrico VI, in cui all'interno di una architettura religiosa, sono appese alcune lampade in vetro, caratterizzate da un'esile fiammella. (Fig. 11) E infine le stesse lampade le ritroviamo ancora in una miniatura dell'Exultet di Cassino Barb. Lat. 592, presso la Biblioteca Apostolica Vaticana, eseguito nell'ultimo quarto dell'XI secolo. (Fig. 12)

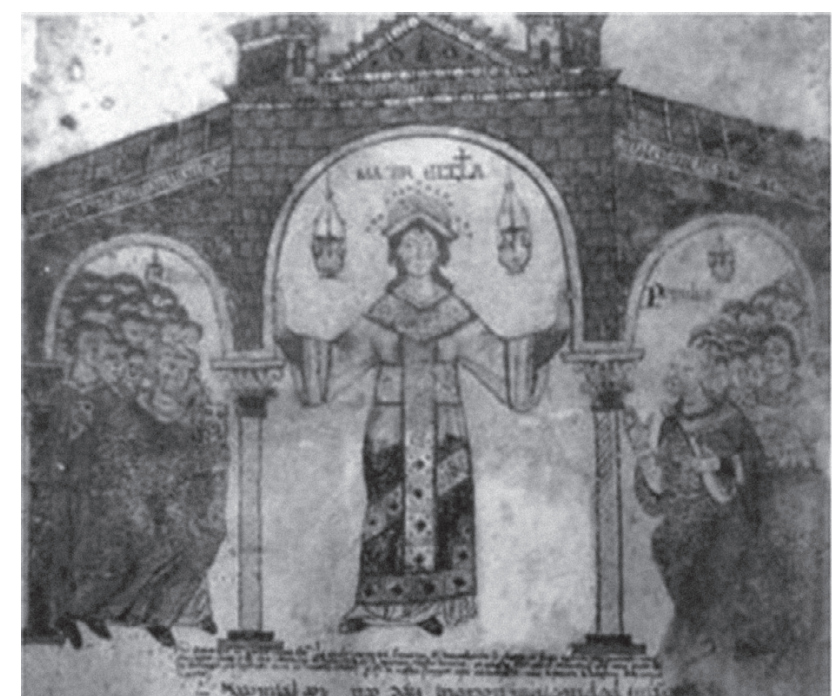

Fig. 12: Miniatura dell'Exultet di Cassino Barb. Lat. 592, presso la Biblioteca Apostolica Vaticana.

Come possiamo ben vedere, tutti i manufatti analizzati sono accomunati dall'esigenza di rimanere sospesi in modo da ottimizzare la loro funzione illuminante in base al luogo in cui erano posti. Necessario a ciò è l'utilizzo di anelli, ganci, distanziali e catene. Va sottolineato che non sembrano documentate suspensurae costituite da sole catene, poiché ogni elemento portante è composto dall'insieme di diversi oggetti che si susseguono tipologicamente dalla tarda antichità al bassomedioevo. Troviamo suspensurae che utilizzano anelli di varia grandezza, congiunti a distanziali rigidi, sagomati a fettuccia o a losanga, e/o distanziali filiformi, uniti a porzioni di catenelle da due o più maglie che con la mediazione di ganci e uncini garantivano l'aggancio stabile della lampada al muro o al soffitto ${ }^{31}$. In particolare, a Cencelle, ritroviamo un esemplare di suspensura metallica con distanziali filiformi, composto da uno o due fili a sezione circolare con estremità piegate ad occhiello della lunghezza di $8 \mathrm{~cm}$ ciascuna e un distanziale centrale più lungo $(14 \mathrm{~cm})$ a cui si agganciano tutti gli altri. (Fig. 13)

Per quanto riguarda questo tipo di manufatti, sono stati documentati inoltre durante gli anni di scavo, oggetti che se messi insieme, possiedono le qualità per tenere sospesa una singola lampada. Sono testimoniate 1 porzione di catenella a due maglie lunga circa $6.5 \mathrm{~cm} ; 2$ distanziali rigidi inseriti 31 M. CORRADO, Sistemi metallici di sospensione e lampade vitree pensili in Calabria dalla tarda antichità al medioevo, in Temporis Signa, IV (2009), pp.
139-169, fig. 2 (a-d). 


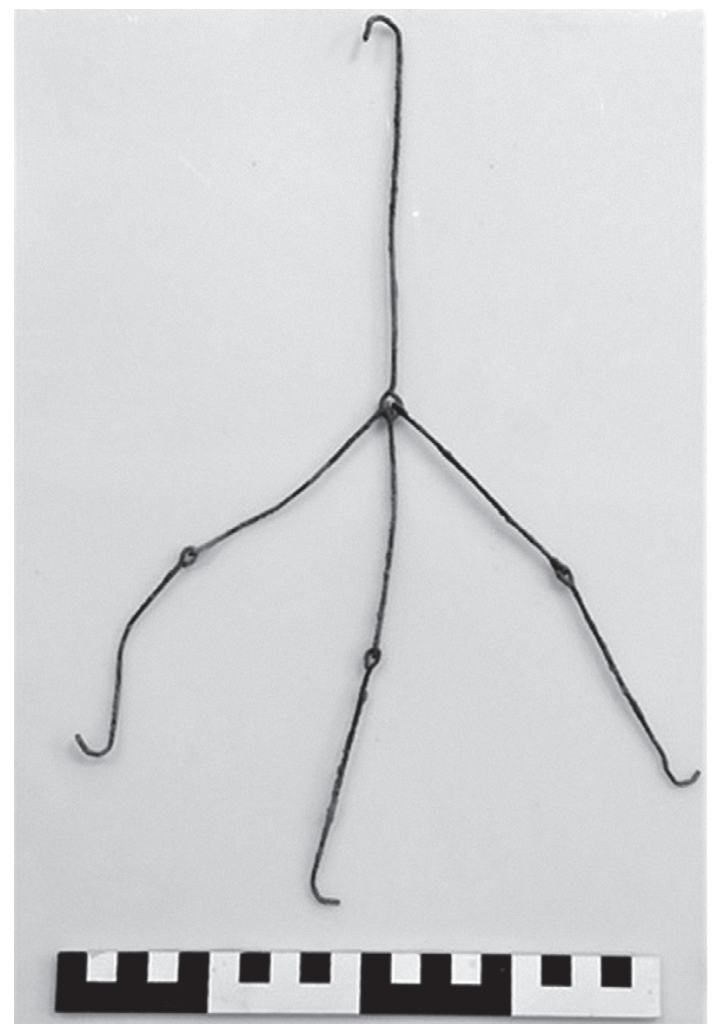

Fig. 13: Suspensura metallica con distanziali fliformi proveniente da Cencelle. Composto da uno o due fili a sezione circolare con estremità piegate ad occhiello e un distanziale centrale più lungo a cui si agganciano tutti gli altri.
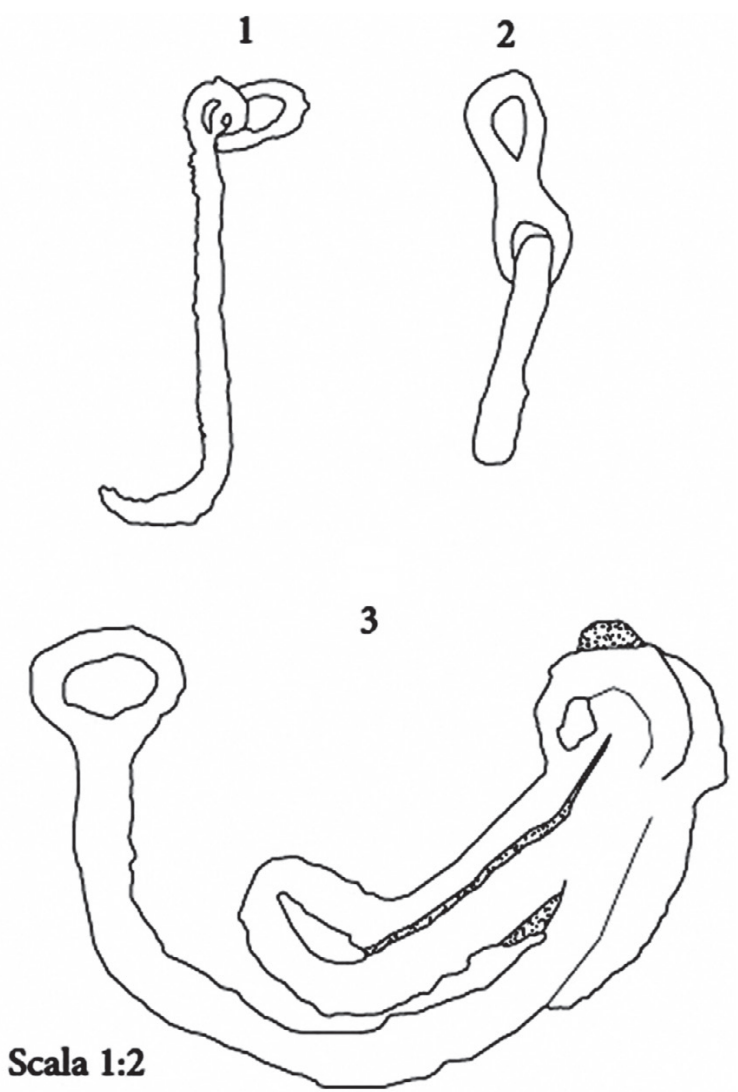

Fig. 15: Ganci e catene per sospensione provenienti da Cencelle: 1. Gancio con piccolo occhiello superiore tramite il quale può legarsi a un altro supporto; 2. Porzione di catenella a due maglie; 3. Oggetto per la sospensione composto da 2 distanziali rigidi inseriti uno dentro l'altro.

\section{Scala 1:1}
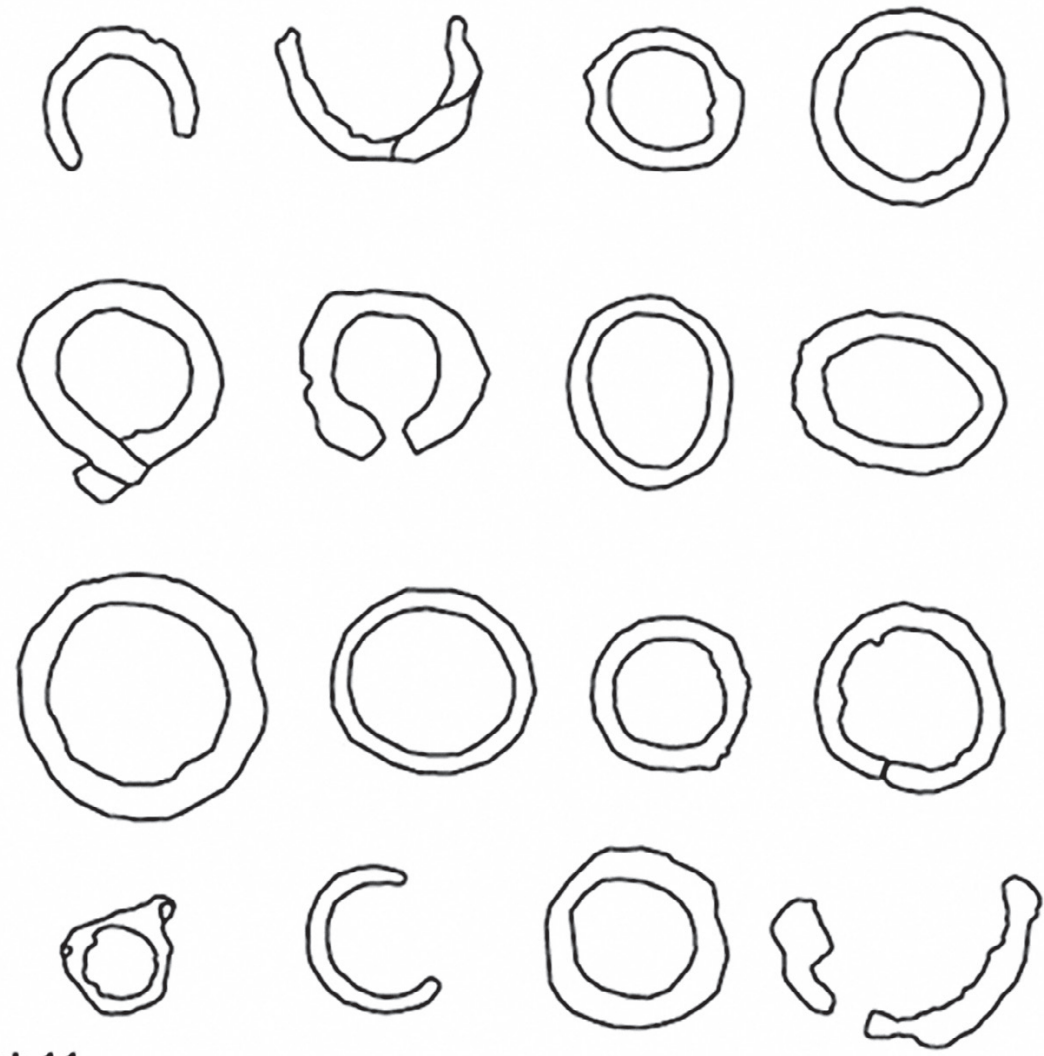

Fig. 14: Anelli regolati provenienti da Cencelle con diametro che oscilla tra 1,5 e $3 \mathrm{~cm}$ e spessore di 0,4/0,5 cm.

uno dentro l'altro, lunghi circa $14 \mathrm{~cm}$; 3 ganci con piccolo occhiello superiore tramite il quale possono legarsi a un altro supporto; 16 anelli regolari con diametro che oscilla tra 1,5 e 3 $\mathrm{cm}$ e spessore di o, $4 / 0,5 \mathrm{~cm}$; 3 anellini regolari con diametro di $1 \mathrm{~cm}$ e spessore di o,6 cm. (Figg. 14-15)

Giulia Previti

L'analisi dei manufatti destinati all'illuminazione a Cencelle mostra una singolare scarsità di resti, in una città che ha restituito un numero di manufatti piuttosto consistente, sia in termini di quantitativi generali, sia in termini percentuali $3^{2}$. Questo dato va in parte imputato alla struuuta stessa dell'abitato, composto nel pieno medioevo prevalentemente da abitazioni di Cencelle a due piani, dove un focolare privo di camino nell'ambiente al piano inferiore doveva essere sufficiente per gli usi di cucina e di illuminazione ${ }^{33}$. Le rare lucerne in ferro e in vetro, oltre certamente alle candele, dovevano servire per lo sporadico uso esterno o ai piani superiori, se necessario. Nuclei abitativi quindi nei quali la vita sociale si concentrava nell'unico ambienteal pian terreno, illuminato dalla porta e dalle finestre quanto a luce naturale, e dal focolare quanto a luce artificiale, una stanza di modeste dimensioni nella quale la mancanza di caminetti favoriva la diffusione della luce del fuoco, che si spandeva a fiamma libera. Diverso è il discorso per le strutture pubbliche, come le sedi del potere, o quelle militari, o ancora quelle religiose, a cui vanno riferiti con ogni probabilità i frammenti di lampade vitree e gli elementi metallici che dovevano essere utilizzati per la loro sospensione. La mancanza di illuminazione pubblica non doveva costituire un problema, a fronte di una

32 Si rimanda a STASOLLA 2018a, op. cit. (nota 2) e STASOLLA 2018b, op. cit. (nota 2).

33 Per l'analisi delle strutture abitative si rimanda ai contributi di E. De Minicis, R. Martorelli, F. Bougard in STASOLLA 2012, op. cit. (n. 1). 
normativa statutaria che scoraggiava in ogni modo la circolazione dopo il tramonto e prima dell'alba, arrivando a proibirla senza una buona ragione. Nell'analisi di questa categoria di manufatti, quindi, ci si trova di fronte ad un'assenza archeologica che può essere in parte spiegata con l'utilizzo di manufatti deperibili, come torce o candele, il cui uso è tradito dalla presenza di portacandele; si tratta di un unico esemplare, ma una candela poteva essere alloggiata anche su manufatti di fortuna non più riconoscibili, come ad esempio piattini o frammenti ceramici o lamine metalliche. Ma l'assenza può essere anche indizio di una mancata presenza, come lasce- rebbero intuire le prescrizioni delle normative statutarie, che prescrivendo di fatto un coprifuoco notturno per ragioni di ordine sociale, limitano l'uso di strumenti di illuminazione nelle città, abbassandone drasticamente il record archeologico. Un dato, questo, che darebbe interessante verificare in modo comparativo in centri di diversa dimensione, o con diverse condizioni di controllo - ad esempio dotati o meno di ronde notturne - per comprendere quanto ed in che modo la presenza di strumenti di illuminazione sia funzionale anche ad attività esterne alle strutture abitative.

Francesca Romana Stasolla 